Construir comunicación en una radio indígena del norte argentino

Entrevista al comunicador comunitario Sebastián Reyes

Silvia Hirsch, Agustín Diz

Tram[p]as de la comunicación y la cultura (N. $\left.{ }^{\circ} 86\right)$, e046, 2021

ISSN 2314-274X | https://doi.org/10.24215/2314274Xe046

http://perio.unlp.edu.ar/ojs/index.php/trampas

FPyCS | Universidad Nacional de La Plata

La Plata | Buenos Aires | Argentina

\title{
CONSTRUIR COMUNICACIÓN EN UNA RADIO INDÍGENA DEL NORTE ARGENTINO
}

\author{
BUILDING COMMUNICATION IN AN INDIGENOUS \\ RADIO IN NORTHERN ARGENTINA \\ Silvia Hirsch \\ silviahirsch5@gmail.com \\ https:// orcid.org/0000-0002-6086-085X \\ Instituto de Altos Estudios Sociales \\ Universidad Nacional de San Martín | Argentina \\ Agustin Diz \\ agustin.diz@ed.ac.uk \\ https://orcid.org/0000-0003-1643-2652 \\ University of Edinburgh | Reino Unido
}

\begin{abstract}
Resumen
Sebastián Reyes fue un indígena chané que a partir de su formación en comunicación comunitaria y periodismo creó programas en Radio Nacional (Tartagal), conformó la Red de Comunicadores Indígenas y creó una radio en la comunidad rural Yacuy, para brindar información y transmitir el acervo cultural, histórico y musical de los pueblos indígenas.
\end{abstract}

\begin{abstract}
Sebastián Reyes was an indigenous Chané who built on his training in community communication and journalism radio programmes for Radio Nacional (Tartagal), formed part of the Network of Indigenous Communicators and created a radio in the rural community of Yacuy, to provide information and transmit knowledge about the cultural, historical and musical heritage of indigenous peoples.
\end{abstract}

Palabras clave | comunicación intercultural, radio comunitaria, indígenas Keywords | intercultural communication, community radio, indigenous people Recibido: 13/10/2020 | Aceptado: 11/12/2020 | Publicado: 03/02/2021 


\section{CONSTRUIR COMUNICACIÓN EN UNA RADIO INDÍGENA DEL NORTE ARGENTINO}

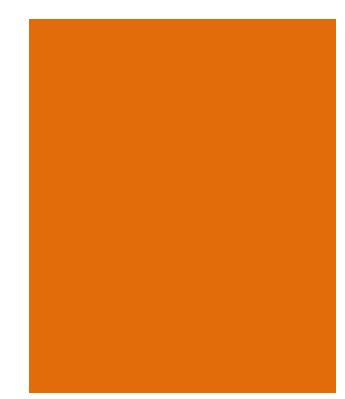

Por Silvia Hirsch y Agustin Diz

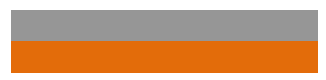

Sebastián Reyes fue un comunicador comunitario e indígena chané que conformó la Red de Comunicadores Indígenas y creó una radio comunitaria en Yacuy, comunidad rural ubicada a $19 \mathrm{~km}$ de la ciudad de Tartagal, Salta. Su objetivo fue generar un espacio para brindar información $\mathrm{y}$ para transmitir conocimientos sobre el acervo cultural, histórico y musical de los pueblos indigenas.

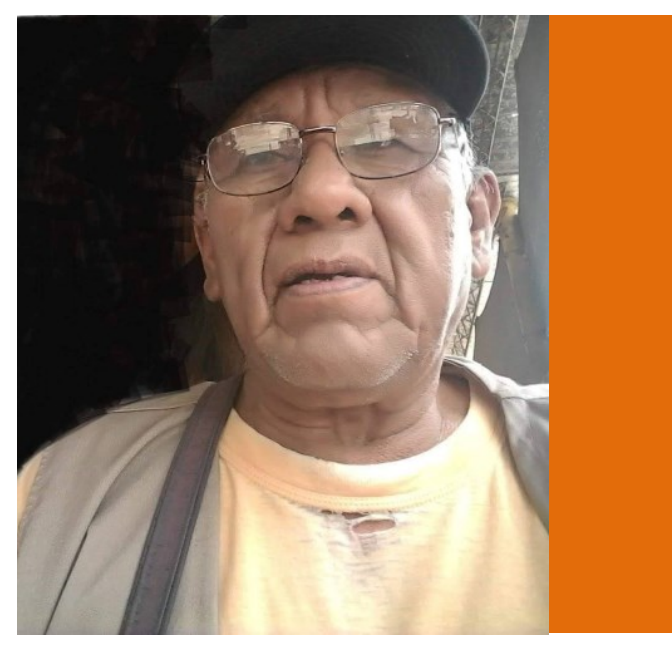

Esta entrevista se realizó en diciembre de 2012. Sebastián falleció en julio de 2019. Quisimos publicarla en homenaje a su memoria y a su lucha por una comunicación indígena y alternativa. 
Una tarde de intenso calor en la comunidad guarani de Yacuy, ubicada en el norte de la provincia de Salta, Argentina, en diciembre de 2012, entrevistamos a Sebastián Reyes, fundador de la Radio FM Kaa Guasu, una radio comunitaria creada con un gran esfuerzo personal, y cuyo objetivo es generar un espacio de difusión y de comunicación de la cultura de los pueblos originarios del norte argentino.

Yacuy es una comunidad rural ubicada a $19 \mathrm{~km}$ al norte de la ciudad de Tartagal, donde viven aproximadamente 2.000 habitantes, muchos de los cuales se dedican a la agricultura y a diversos trabajos estacionales $y$ permanentes en la zona. Al caminar por las calles de Yacuy, se observa una numerosa presencia de niños que hablan en su lengua materna. En la escuela de la comunidad, hace casi dos décadas que funciona un proyecto de Educación Intercultural Bilingüe, que fortalece la lengua y la cultura de la comunidad y que ha permitido incorporar a varios/as jóvenes como docentes bilingües.

La radio es un espacio de difusión de ideas, de música y de mensajes entre las diversas comunidades de la zona, lo que contribuye a generar un espacio alternativo a los medios de comunicación dominantes. Quienes participan en la radio eligen la programación de su interés, la cual varia de programas de música evangélica, a programas sobre problemas de tierras o mensajes informativos que comunican a gente de parajes y de comunidades distantes.

Sebastián Reyes, quien hace varias décadas se dedica al periodismo radial y comunitario, y que tuvo la idea de fundar esta radio, nos habló de su trayectoria como periodista y comunicador social. Para él, la radio genera un espacio de inclusión, en el cual no se margina a quienes quieran participar, como locutores o para desarrollar programas, simplemente por no tener experiencia o por pertenecer a un grupo minoritario. FM Kaa Guasu es una radio abierta a la participación de jóvenes, de niños y de mayores, abierta para quienes quieran dar a conocer sus inquietudes y sus conocimientos más allá de su propia comunidad. 


\section{Hablemos de su trayectoria, ¿Cuándo comenzó a interesarse por el periodismo?}

Siempre tuve en mi mente hacer radio. Me acuerdo cuando vivía en Cornejo, ${ }^{1}$ allá por 1960. En esos tiempos, estaban Radio El Mundo, Radio Splendid, Radio Mitre... En esa época (habré tenido diez años, más o menos), escuchaba los partidos de fútbol que Fioravanti2 transmitía por Radio El mundo. Desde chico, eso me gustó bastante, y muchas veces lo imitaba.

Pasaron muchísimos años, y recuerdo que en 1975 comencé a mirar: había una sola radio en Tartagal. Hice comunicación en la Iglesia, donde preparábamos (especialmente, en tiempos de Navidad) una escena para representar la vida de Jesús en la que leíamos la Biblia en forma de radio. En 1980, me vine a vivir a Yacuy. Para uno de los días de la celebración de la Pascua venían los hermanos de Bolivia y traian casetes grabados. Eso me interesó bastante y se me dio la oportunidad de hacer un programa de radio.

En 1985 o 1986, di los primeros pasos para poder salir con una radio AM comercial. Hablé con el director, que era el dueño, don Vicente Pardo, pero me pedian un montón de requisitos y como no podia conseguir todo lo que me pedian (entre ellos, un currículo), pensé: "¿Qué hago?" Y me fui a Radio Nacional. Estaba allí su director, don Víctor Sedan, y le dije: "Don Víctor", "¿Quién sos?", me contestó, "Sebastián Reyes, de la comunidad de Yacuy", me presenté.

—Qué lindo que venís, pasá. ¿Qué te trae por acá?

- Me trae esta inquietud. Tengo una visión de hacer un programa de radio, con un formato especialmente dedicado a los pueblos originarios, a los pueblos indígenas, guaraní.

- Te doy 15 minutos los días martes y 15 minutos los días jueves.

Entonces, fui e hice los programas. Me senté y parecía que tenía experiencia. Un operador técnico me preguntó: “¿Cuál es el formato que tenés?». Entonces, hice la presentación del programa y lo llamé "Historia de nuestro pueblo". 
Se trataba de contar la historia de nuestro pueblo argentino-guaraní. En ese tiempo, teníamos contacto con los guaraníes de Bolivia y de ahí nació la idea de hacer un programa, porque ya 15 minutos era poco, pero fueron los primeros pasos. Fue el 2 de abril de 1980 cuando me pregunté: "¿Cómo tengo que hacer la comunicación?". Lo primero que pensé es cómo hacemos nosotros comunicación, en el hogar, en el campo, en la ciudad, en la cacería, con el canto de los pájaros o con el ruido del agua. Cuando vas a la playa, por ejemplo, $\mathrm{y}$ ves que queda algún vestigio de agua, tenés que quedarte calladito a ver a dónde va ese cauce de agua. Me acordaba de todo eso y empecé a aplicar ese método del pueblo guaraní.

Estaba haciendo comunicación, entonces, según nuestra experiencia de cómo hacíamos para comunicarnos, para vivir dentro de nuestra comunidad. Por ejemplo, cómo nos comunicábamos cuando íbamos de cacería. A veces éramos tres y nos separábamos en el bosque. Como es un bosque grande, en el que uno se puede perder, para volvernos a encontrar marcábamos con un palo o hacíamos una flecha por donde habíamos ido, y si la persona llegaba a esa flecha hacía otra indicando dónde nos íbamos a encontrar. Así, íbamos hacia donde la flecha nos indicaba y sabiamos que el otro iba a estar ahí.

Los años ochenta fueron duros, pero pasamos buenos y malos momentos. Cuando vine no tenía recursos, nada de nada (había un Instituto Provincial del Aborigen -ahora denominado Instituto Provincial de Pueblos Indígenas-, pero era un nombre y sigue siendo un nombre. Nunca me aferré a ellos y he estado con amigos de ONG que me conocen y he sido constante en mi trabajo y con mi familia, sobre todo, así que tengo para vivir). Todos los martes y los jueves, me iba en bicicleta unos $20 \mathrm{~km}$. Para no venir sin nada de plata, ponía en la bicicleta un cajón de tomate y en la ruta juntaba todo lo que era aluminio para luego vender a los gitanos.

A veces, me tocó venir a pie. Recuerdo un día, casi un 24 de diciembre, ya finalizando los programas de radio, que me tocó venir a pie, pero cumplí mi objetivo. Luego de eso empezamos a hacer proyectos, proyectos a futuro. Para 1990 teníamos una pequeña beca 
de ENDEPA (Equipo Nacional de Pastoral Aborigen) para poder movernos. También conseguimos una grabadora para salir al campo a hacer entrevistas que me sirvió bastantes años. No fue una herramienta para escuchar música solamente, era para grabar noticias de la comunidad, para contar por qué están viviendo así, qué hay que decirles a los dirigentes, al gobierno de la provincia, al intendente, por ejemplo. Y así fueron los programas en Radio Nacional, programas con contenido.

Para hacer el programa, elegí las tres de la tarde porque es la hora en la que las mujeres regresan de vender el producto agrícola en las ciudades vecinas y la gente descansa en sus casas antes de volver otra vez al campo. Por eso elegí esa hora para nombrar toda la historia de nuestro pueblo.

Pasados esos años, fue el tiempo de estudiar la reforma de la Constitución Nacional. Fuimos a Buenos Aires e hicimos un seminario para tener conocimiento de qué era. No sabíamos que decía la Constitución, ni sabíamos lo que era una Constitución. Así fue como nos formamos, siete provincias que estábamos éramos guaraní, tobas, coyas y también mapuches. De esa manera, fuimos conociendo lo que era, que garantías teniamos. Y encontramos que en la vieja Constitución no había garantías para nosotros. Digamos que el gobierno le decía al cura, al religioso, que vaya a buscar indios a la frontera para convertirlos al catolicismo. Nos dimos cuenta que el gobierno tenía bastante miedo de nosotros, los indigenas. Cuando hablamos de la campaña al desierto decimos que a nosotros nos tenian que convertir al catolicismo para que seamos mansos, pero no era tan así. Teníamos que tener todos los derechos: educación, salud, agua potable. La comunidad tenía que tener su título de tierra, que fuera apta y suficiente. Los gobiernos nos tenían que reconocer... Y así fue.

En 1992 se cumplieron en Bolivia 100 años de la batalla de Kuruyuki, ${ }^{3}$ cuando los hacendados quisieron hurtar todo el territorio guaraní, y me invitaron como periodista. Me reconocieron como periodista en asuntos indigenas y me dieron la oportunidad de aprender mejor a hablar guaraní, así que me interné a estudiar 
durante un año. Nos juntamos en Kuruyuki. Mucha gente, llegaron casi 6.000 guaraníes. Nosotros como argentinos nos juntamos con los sectores de Bolivia y de Paraguay. Y ahí nos encontramos.

En 1994 fue la reforma de la Constitución Nacional y teníamos que estar presentes. Fue un desafio muy grande como periodistas. Si no tenías acreditación del gobierno, no eras periodista, nadie te iba a creer que eras periodista, pero yo me preparé, estudié en INCUPO, 4 en Reconquista; siempre lo reconozco porque salí de ahí. Tenía mi carnet de acreditación, nos decian "los legisladores de pasillo". Yo llevaba la última noticia a los indígenas. A veces eran la una o las dos de la madrugada y no había terminado de relatar lo que se había hablado ese día.

Cuando fuimos a la Reforma de la Constitución, el 11 de agosto de 1994, cumplimos nuestro objetivo: hablar por la radio sobre nuestros derechos, porque tenemos derechos, a educación, a salud... Ese día, Argentina lo reconoció por amplia mayoría, por unanimidad. En el artículo 75 , inciso 17 , se escribió que somos pluricultural. ${ }^{5}$ También hay que ver otros derechos en lo internacional, como el Convenio 169 (de la OIT). ${ }^{6}$ Todos esos derechos eran para tener argumentos para discutir cosas grandes en la radio. Empezamos a ver el tema del territorio, cómo hacer, dónde buscar cartillas con información sobre nuestros derechos.

Después llegaron los desmontes: la soja. Hay que preguntar a los legisladores por qué cuando pasan los aviones cerca de tu casa hay mucha desnutrición, contaminación. Estuve metido en ese tema, tuve contacto con ambientalistas. Todo eso que a un periodista le hace falta para tener argumentos. Ahora, por ejemplo, con el cambio climático, ¿por qué el cambio climático?

Si hay desmonte, llueve y se inunda. Todas esas cosas que hoy estamos pagando nosotros. Por eso, como cuidadores de bosques, ya empezamos a armar una organización transnacional con Bolivia, Argentina y Paraguay, hablando sobre los derechos de los pueblos indígenas, sobre la autonomía de los pueblos, y ya empecé a prepararme para conocer la politica y defenderlos. 


\section{¿Cómo se incorporó a la Red de Comunicadores Indigenas?}

En 2000, nació en Pampa del Indio, provincia de Chaco, la Red de Comunicadores Indígenas para cinco provincias de NEA y de NOA.7 Conseguimos que en Alemania nos reconocieran como periodistas indígenas, también a través de INCUPO, que fue nuestro vocero y nos apoyó para formar la red.

He sido referente para Salta y Jujuy. Una de mis compañeras trabajó para Radio Nacional, también sin ser de la radio, aficionados nomás, pero logramos bastante. Armamos la Red de Comunicadores y logramos tener 60 periodistas indigenas, en NEA y NOA. Caritas de Friburgo, Alemania, nos apoyó por diez años, por lo que tenemos un equipo muy importante y una mesa directiva para que la Red tenga su propia politica de comunicación. Luego, salió para el NOA la posibilidad de tener otro estudio de grabación, y los hermanos de Jujuy me eligieron para trabaje en esa radio, así que otra vez tuve que ir a vivir a Jujuy. Pasaron diez años y logramos un estudio tal cual están viendo mi radio, más grande, pero el modelo era así.

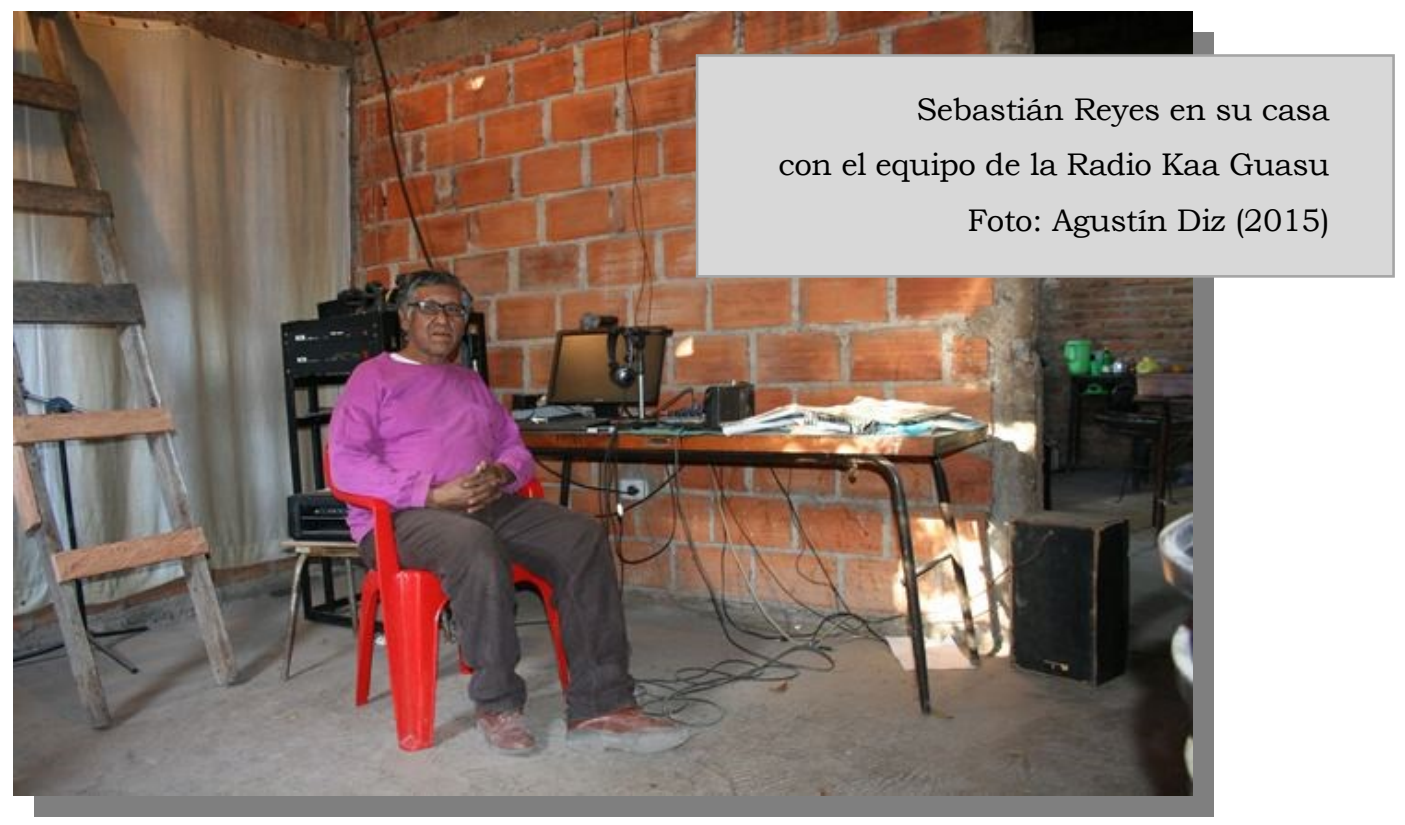

En Jujuy teníamos el segundo estudio, donde haciamos programas, grabábamos discos, envasábamos. Ahí aprendí sobre la cibernética. Tuvimos 17 antenas satelitales, lo que nos permitió difundir los programas. Les dije que tenía un programa por la radio, que tenía mi 
trayectoria, y que estaba dispuesto a acompañarlos. Pero también había que pensar que el día de mañana eso se terminaba y que tenía que volver a mi comunidad. Así que tuve que pensar en comprarme una radio, que la tengo en $\mathrm{mi}$ casa, porque la radio es la voz del pueblo, para saber qué es lo que está pasando, en guaraní, en castellano. Ahora, hace un año y medio que estoy en Yacuy.

Me fui a trabajar en mi campo. Tengo mi campo para sembrar y además soy artesano, hago máscaras. Con mi hijo José Luis tratamos de arreglarnos, vamos al campo. Decir que estuve once años en Jujuy no quiere decir que me va a cambiar todo. Tengo fuerza, tengo bicicleta, tengo mi campo. Pensé: "Ya me vengo, no quiero seguir cuidando cosas ajenas. Estuve mucho tiempo allá, ya cumplí mi sueño".

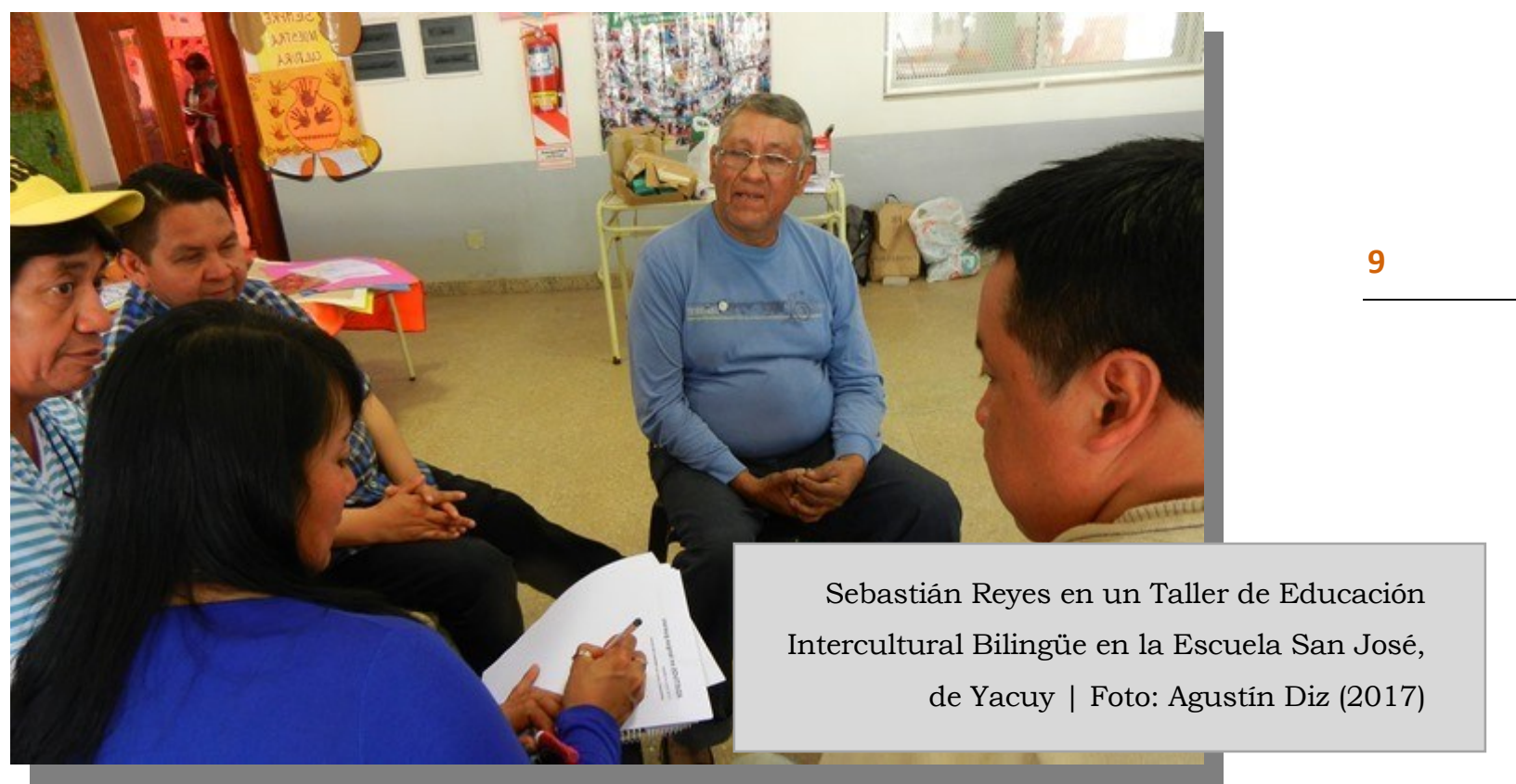

Hay equipos que están armados artesanalmente, porque sacan de otros. La máquina que están viendo es un AM 23; estamos llegando a $50 \mathrm{~km}$ alrededor, que fue mi gran objetivo. El 26 de marzo, va a cumplir un año de emisión y tenemos auspiciantes. A la familia que le gusta hacer el programa. Yo me dediqué a poner un vivero, cerca de la escuela, así que vendo picolé, ${ }^{8}$ gaseosa. Tengo para pagar la luz todos los meses, no tengo drama. Ahora estamos tratando de que los 21 puntos, lo que nosotros hicimos por la autorización federal, 
la nueva Ley 26.522,9 se cumpla y así firmar para que en un futuro esta radio nos nombre Kaa Guasu. ¿Por qué? Porque quiere decir monte grande. Esta es la única selva chaqueña que está quedando... Ojalá que nadie lo desmonte.

A ese gran Kaa Guasu que tenemos, a ese monte grande, queremos mantenerlo y ser nosotros la voz. Tenemos como lema ser la luz que señala el camino de la vida, que es el cuidado del bosque. El bosque nos da medicina provisional, podemos descansar, nos cobija en la sombra, nos protege de la lluvia. Nos cobija de todo: el Kaa. ${ }^{10}$

\section{¿Qué tipo de programas hacen?}

En Yacuy hay más de 2.000 habitantes y la radio está a cuatro cuadras de la ruta nacional, a $20 \mathrm{~km}$ de la ciudad de Tartagal. Como es la única radio en el medio de dos ciudades, nosotros somos el nexo para muchos. Tenemos señal y oyentes de Fátima, Zanja Honda y hasta Cornejo, que se comunican, mandan mensajes.

Arranco a las 8 de la mañana con el editorial de alguna revista indigena. Cuando comienzan las vacaciones ponemos música autóctona, el "pin pin", con música variada. Hay varios bloques: tenemos un bloque de noticias, después un bloque musical, hay de todo. A las 21 tenemos hora de tango, para los amigos taxistas y para los trasportistas que vienen de Santa Fe o de Córdoba (que me dicen: "¡Che, poné tango!»). También hay un bloque evangélico y otro católico. Yo conozco a los indígenas de Chiapas, en México, y en honor al pueblo mexicano tengo un programa que se llama "México canta a la distancia".

Tengo que demostrar que todavía tengo capacidad para hablar y sigo hablando como un locutor de radio. Ahora tengo programación: los jóvenes hacen un programa, Amado Soria hace otro que se llama "Nuestro canto, nuestra tierra y nuestra gente». Lo puse porque habla mucho del tema de la tierra, los plantines, cosas de nuestra gente. 
Por la noche hacen programas los jóvenes. Los que vienen hacen locución y a la vez hacen de operadores; a veces, uno habla y el otro hace de técnico. Con un coraje... Me encanta verlos, que la radio sea para los chiquitos y hasta los de 70 , todos pueden venir a hablar. No quiero que la radio margine a nadie, porque yo lo he vivido. En la primera radio AM me cerraron la puerta y yo no quiero eso para mi pueblo, para ningún pueblo. Quiero que todos tengan derecho a hablar, a cantar como el pájaro que es libre, autónomo.

La radio es una herramienta que cualquiera puede usar, para venir a hablar. La radio trae noticias, avisos, comunicados. Por ejemplo: "Atención Yacuy, hoy hay una reunión en el centro comunitario. A las 15.30 se trata de la tierra", o de otra cosa. O me dicen que necesitan leña y acá hay un leñero, que es de Fátima, yo aviso: "Atención don Demetrio, necesitamos dos viajes de leña". A veces, la radio es un servicio para la gente, sirve para eso. Si la persona quiere comunicarse, dejar un mensajito, la radio le da esa posibilidad.

Sebastián no pudo continuar con su sueño y su gran pasión: no consiguió la licencia requerida y no pudo sostener económicamente la radio. Pero será recordado, más allá de su comunidad, como un gran comunicador, como un hombre que logró darles un espacio a voces largamente silenciadas.

\section{Notas}

1 Localidad argentina del Departamento General José de San Martín, provincia de Salta, ubicada sobre la Ruta Nacional 34.

2 Fioravanti es el nombre profesional de Joaquín Carballo Serantes (19111989), un relator radial de fútbol que trabajó en Radio Splendid y en Radio E1 Mundo.

3 La Batalla de Kuruyuki o Batalla de Curuyuqui se libró en las proximidades de la ciudad de Cuevo, Bolivia, el 28 de enero de 1892.

4 El Instituto de Cultura Popular (INCUPO) es una organización de la sociedad civil que trabaja con organizaciones campesinas e indigenas. 
5 El mencionado inciso, indica: "Reconocer la preexistencia étnica y cultural de los pueblos indígenas argentinos. Garantizar el respeto a su identidad y el derecho a una educación bilingüe e intercultural; reconocer la personería Jurídica de sus comunidades, y la posesión y propiedad comunitarias de las tierras que tradicionalmente ocupan; y regular la entrega de otras aptas y suficientes para el desarrollo humano; ninguna de ellas será enajenable, transmisible ni susceptible de gravámenes o embargos. Asegurar su participación en la gestión referida a sus recursos naturales y a los demás intereses que los afecten. Las provincias pueden ejercer concurrentemente estas atribuciones".

6 El Convenio 169 de la Organización Internacional del Trabajo (OIT) sobre pueblos indígenas y tribales es una convención adoptada en 1989 que constituye el principal instrumento internacional sobre derechos de los pueblos indigenas.

$7 \mathrm{El}$ nordeste argentino (NEA) es una región que comprende las provincias de Formosa, Chaco, Corrientes y Misiones. El noroeste argentino (NOA) está compuesto por las provincias de Jujuy, Salta, Tucumán, Catamarca, La Rioja y Santiago del Estero.

8 La paleta o picolé es un helado hecho a base de agua, colorante, saborizante y azúcar, de forma alargada y con un palo que lo atraviesa para tomarlo.

9 Ley de Servicios de Comunicación Audiovisual, sancionada en octubre de 2009. Recuperado de

http://servicios.infoleg.gob.ar/infolegInternet/anexos/155000159999/158649/norma.htm

10 Nos cobija el Kaa, significa que el monte brinda protección, aporta animales para cazar, frutos del monte, y es un lugar de espiritualidad. 